\title{
Correction to: The effect of exosomes derived from mesenchymal stem cells in the treatment of induced type 1 diabetes mellitus in rats
}

\author{
Dina Sabry (D) Samar Marzouk - Reem Zakaria - Heba A. Ibrahim • \\ Mai Samir
}

Published online: 10 August 2020

(C) Springer Nature B.V. 2020

Correction to: Biotechnology Letters (2020) 42:1597-1610

https://doi.org/10.1007/s10529-020-02908-y

In the original publication of the article, the reference citation style in the article was published incorrectly. The journal follows 'Name and Year' style for references. However, they were cited in numbering style incoherent to the references given in the Reference section which were placed in alphabetical order.

The correct Reference section is given below.

\section{References}

1. Copenhaver M, Hoffman R (2017) Type 1 diabetes: where are we in 2017? Transl Pediatr 6:359-364

The original article can be found online at https:// doi.org/10.1007/s 10529-020-02908-y.

D. Sabry $(\bowtie) \cdot$ S. Marzouk $\cdot$ R. Zakaria $\cdot$ M. Samir Medical Biochemistry and Molecular Biology, Faculty of Medicine, Cairo University, Giza, Egypt

e-mail: dinasabdry@kasralainy.edu.eg

H. A. Ibrahim

Pathology Department, Faculty of Medicine, Cairo

University, Giza, Egypt
2. Chen Q, Shou P, Zheng C, Jiang M, Cao G, Yang Q, Cao J, Xie N, Velletri T, Zhang X, Xu C, Zhang L, Yang H, Hou J, Wang Y, Shi Y (2016) Fate decision of mesenchymal stem cells: adipocytes or osteoblasts. Cell Death Differ 23:1128-1139

3. Gnecchi M, Danieli P, Malpasso G, Ciuffreda M (2016) Paracrine mechanisms of mesenchymal stem cells in tissue repair. Methods Mol Biol 1416:123-146

4. Stahl P, Raposo G (2018) Exosomes and extracellular vesicles: the path forward. Essays Biochem 62:119-124

5. Lawson C, Vicencio J, Yellon D, Derek S, Davidson S (2016) Micro-vesicles and exosomes: new players in metabolic and cardiovascular disease. $\mathrm{J}$ Endocrinol 228:57-71

6. Akbarzadeh A, Norouzian D, Mehrabi M, Jamshidi S, Farhangi I, Verdi I, Mofidian S, Rad B (2007) Induction of diabetes by streptozotocin in rats. Indian $\mathrm{J}$ Clin Biochem 22:60-64

7. Wang M, Liang C, Hu H, Zhou L, Xu B, Wang X, Han Y, Nie Y, Jia S, Liang J, Wuet K. (2016). Intraperitoneal injection (IP), Intravenous injection (IV) or anal injection (AI) Best way for mesenchymal stem cells transplantation for colitis. Sci Rep, 6: ID 30696.

8. Wu Y, Deng W, Klinke D (2015) Exosomes: improved methods to characterize their morphology, RNA content, and surface protein biomarkers. Anal 140:6631-6642

9. Braun R, Chetty C, Balasubramaniam V, Centanni R, Haraldsdottir K, Hematti P, Eldridge M (2018) Intraperitoneal injection of MSC-derived exosomes prevents experimental bronchopulmonary dysplasia. Biochem Biophys Res Commun 503:2653-2658

10. Abdel AM, El-Asmar M, Haidara M, Atta H, Roshdy N, Rashed L, Sabry D, Youssef M, Abdel AA, Moustafa M (2008) Effect of bone marrow-derived mesenchymal stem cells on cardiovascular complications in diabetic rats. Med Sci Monit 14:249-255

11. Bruno S, Grange C, Deregibus M, Calogero R, Saviozzi S, Collino F, Morando L, Busca A, Falda M, Bussolati B, Tetta 
C, Camussi G (2009) Mesenchymal stem cell-derived microvesicles protect against acute tubular injury. J Am Soc Nephrol 20:1053-1067

12. Chan Y (2003) Biostatistics 102: quantitative data parametric and non-parametric tests. Singapore Med J 44:391-396

13. Katuchova J, Harvanova D, Spakova T, Kalanin R, Farkas D, Durny P, Rosocha J, Radonak J, Petrovic D, Siniscalco D, Qi M, Novak M, Kruzliak P (2015) Mesenchymal stem cells in the treatment of type 1 diabetes mellitus. Endocr Pathol 26:95-103

14. Aghajani A, Lerman L, Eirin A (2017) Mesenchymal stem cell-derived extracellular vesicles for kidney repair: current status and looming challenges. Stem Cell Res Ther 8:273

15 Pinheiro A, Silva A, Teixeira J, Goncalves R, Almeida M, Barbosa M, Santos S (2018) Extracellular vesicles: intelligent delivery strategies for therapeutic applications. J. Control Release 289:56-69

16. Kobolak J, Dinnyes A, Memic A, Khademhosseini A, Mobasheri A (2016) Mesenchymal stem cells: identification, phenotypic characterization, biological properties and potential for regenerative medicine through biomaterial micro-engineering of their niche. Methods 99:62-68

17. De la Torre Gomez C, Goreham R, Bech SJ, Nann T, Kussmann M (2018) Exosomics-a review of biophysics, biology and biochemistry of exosomes with a focus on human breast milk. Front genet 9:92

18. El Barky A, Ezz A, Alm-Eldeen A, Hussein S, Hafez Y, Mohamed T (2018) Can stem cells ameliorate the pancreatic damage induced by streptozotocin in rats. Can J Diabetes 42:61-70

19. Carlsson P, Schwarcz E, Korsgren O, Le Blanc K (2015) Preserved beta-cell function in type 1 diabetes by mesenchymal stromal cells. Diabetes 64:587-592

20. Sun Y, Shi H, Yin S, Ji C, Zhang X, Zhang B, Wu P, Shi Y, Mao F, Yan Y, Xu W, Qian H (2018) Human mesenchymal stem cell derived exosomes alleviate type 2 diabetes mellitus by reversing peripheral insulin resistance and relieving beta-cell destruction. ACS Nano 12:7613-7628
21. Rani S, Ryan A, Griffin M, Ritter T (2015) Mesenchymal stem cell-derived extracellular vesicles: toward cell-free therapeutic applications. Mol Ther 23:812-823

22. Horbelt D, Denkis A, Knaus P (2012) A portrait of transforming growth factor $\beta$ superfamily signaling: background matters. Int J Biochem Cell Biol 44:469-474

23. Macias M, Martin-Malpartida P, Massague J (2015) Structural determinants of Smad function in TGF-beta signaling. Trends Biochem Sci 40:296-308

24. Rutter G, Pullen T, Hodson D, Sanchez A (2015) Pancreatic $\beta$-cell identity, glucose sensing and the control of insulin secretion. Biochem J 466:203-218

25. David C, Massagué J (2018) Contextual determinants of TGF $\beta$ action in development, immunity and cancer. Nat Rev Mol Cell Biol 19:419-435

26. Nojehdehi S, Soudi S, Hesampour A, Rasouli S, Soleimani M, Hashemi S (2018) Immunomodulatory effects of mesenchymal stem cell-derived exosomes on experimental type-1 autoimmune diabetes. J Cell Biochem 119:9433-9443

27. De Frutos C, Webster D, Fahrenkrug S, Fahrenkrug S, Carlson D (2015) 240 precise genome editing of PDX1 by direct injection of transcription activator-like effector nuclease (TALENS) into parthenogenetic pig embryos. Reprod Fertil Dev 28:252

28. Fujimoto K, Polonsky K (2009) Pdx 1 and other factors that regulate pancreatic $\beta$-cell survival. Diabetes Obes Metab 11:30-37

29. Rhee M, Lee S, Kim J, Ham D, Park H, Yang H, Shin J, Cho J, Kim Y, Youn B, Sul H, Yoon K (2016) Preadipocyte factor 1 induces pancreatic ductal cell differentiation into insulin-producing cells. Sci Rep 6:23960

Publisher's Note Springer Nature remains neutral with regard to jurisdictional claims in published maps and institutional affiliations. 